
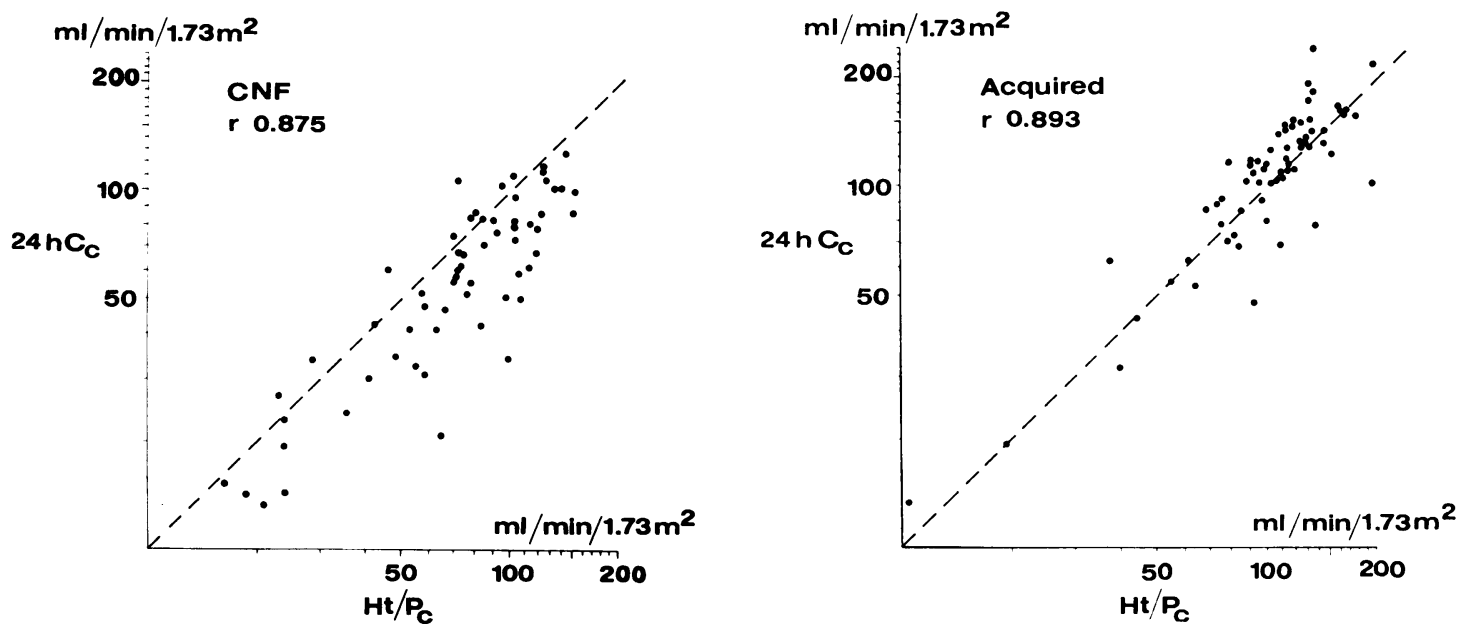

Fig. Correlation between 24-hour endogenous creatinine clearance (24-h $\left.C_{c}\right)$ and glomerular filtration rate estimated by the body height-plasma creatinine concentration method $\left(H t / P_{c}\right)$ in children with congenital nephrotic syndrome $(C N F)$ and in children with acquired renal disease.

type. Scandinavian Journal of Ciinical and Laboratory Investigation, 37, 133-138.

Szelid, ZS., and Méhes, K.(1977). Estimation of glomerular filtration rate from plasma creatinine concentration in children of various ages. Archives of Disease in Childhood, 52, 669-670.

Nillo-Pekka Huttunen, Maisa TaAlikka, and Ritva Metsola

Department of Paediatrics, University of Oulu, and

Children's Hospital, University of Helsinki, Finland.

\section{Nystatin resistance in thrush}

Sir,

Dr R. F. Jennison, in his useful review of thrush in infancy (Archives, 1977, 52, 747), did not mention the occurrence of nystatin resistance. In a recent case I was so impressed by the failure of nystatin, applied by an intelligent mother, to clear the perineal and oral thrush that I sent the baby with his thrush to the laboratory at the Sheffield Children's Hospital to have a culture made. There was a growth of Candida albicans resistant to nystatin (MIC $100 \mathrm{U} / \mathrm{ml}$ ), but sensitive to amphotericin B $(\mathrm{MIC}<\mathbf{0} \cdot 2 \mu \mathrm{g} / \mathrm{ml})$. The thrush cleared promptly with amphotericin.

R. S. ILLINGWORTH 8 Harley Road, Sheffield S11 9SD.

\section{European Congress of Obstetric Anaesthesia and Analgesia}

Sir,

It is intended to hold the first of what is hoped will become a sequence of congresses of this type at the National Exhibition Centre, Birmingham, England, in September 1979. The programme of the congress is designed to be of interest to anaesthetists, midwives, obstetricians, and paediatricians. Its major objective is to provide a forum whereby representatives of these specialities can discuss problems of mutual interest in respect to standards of safety and effectiveness of clinical practice, training, and staffing.

The congress will last for 4 days, and the general titles of the topics to be discussed are: (i) pain relief during labour; (ii) identification of, and supportive therapy for, the fetus at risk; (iii) anaesthesia for operative delivery; (iv) neonatal resuscitation; (v) intensive care of the seriously ill neonate; (vi) intensive care of the seriously ill parturient.

The meetings are being devised in such a way as to encourage the active participation of all delegates in th discussions which will follow each formal presentation. The prepared papers will be presented by anaesthetists, midwives, obstetricians, and paediatricians who are currently in practice in Europe. Simultaneous translation into English, French, and German will be available. A large trade exhibition will be sited within the congress hall, which will be of interest to all delegates. There will also be a full calendar of social events each day for delegates' nonparticipating companions and each evening for the entire company.

More detailed announcements will be forthcoming during the next few months. This preliminary notification is to enable interested readers to make a note of the dates (probably 18-21 September 1979) and, if they so wish, to write to me personally to request advance notice of the details of registration.

J. Selwyn Crawford

Chairman, Organising Committee, ECOAA The Birmingham Maternity Hospital, Queen Elizabeth Medical Centre, Edgbaston, Birmingham B15 2TG. 